count, glucose, proteins and blood leukocyte and differential count, CRP, PCT were studied at the time of suspected ventriculitis. CD64in was measured by flow cytometry (Trillium Diagnostics, LLC, Brewer, ME). Wilcoxon-test was used for comparison between groups and diagnostic accuracy determined by the area under the ROC curves (AUC) was defined for each marker.

Results Thirty-three episodes of clinically suspected ventriculitis in twenty-one children (male 14, female 7, median age: 9 months, range: 8 days-167 months) were observed during a 26month period. Episodes were classified into those with microbiologically proven ventriculitis (13 episodes: 9 Gram-positive and 4 Gram-negative) and those with microbiologically negative CSF (20 episodes). CD64in was the only CSF marker that could differentiate between groups $(\mathrm{p}=0.0003)$; its diagnostic accuracy was 0.875 (95\% CI: 0.713-0.963). Among blood markers only $\mathrm{CRP}$ and band neutrophils differentiated between groups ( $\mathrm{p}=$ 0.0032 and $\mathrm{p}=0.0463)$ with their diagnostic accuracy of 0.808 (0.633-0.923) and 0.721 (0.524-0.870); respectively.

Conclusions CD64in in CSF is a promising diagnostic marker of bacterial ventriculitis in children with external ventricular drainage before microbiological confirmation.

\section{PO-0342 CAN PHOTOPLETHYSMOGRAPHY BE USED TO AUTOMATE CAPILLARY REFILL TIME? PRELIMINARY RESULTS FROM HEALTHY CHILDREN}

${ }^{1} \mathrm{C}$ Henry, ${ }^{1} \mathrm{~S}$ Hill, ${ }^{2} \mathrm{D}$ Morris, ${ }^{2} \mathrm{~L}$ Blaxter, ${ }^{2} \mathrm{~J}$ Crowe, ${ }^{2} \mathrm{~B}$ Hayes-Gill, ${ }^{1} \mathrm{H}$ Vyas, ${ }^{1} \mathrm{D}$ Sharkey. ${ }^{1}$ Division of Child Health Obstetrics and Gynaecology, University of Nottingham, Nottingham, UK; ${ }^{2}$ Faculty of Engineering, University of Nottingham, Nottingham, UK

\subsection{6/archdischild-2014-307384.990}

Background Capillary refill time (CRT) is widely used in paediatrics to assess cardiovascular status, especially during the early phase of shock when skin perfusion is reduced, but is prone to marked variability (Pickard 2011). CRT is used in early warning scores aimed at identifying and quantifying severity of illness

\section{Green light PPG CRT trough and peak values}

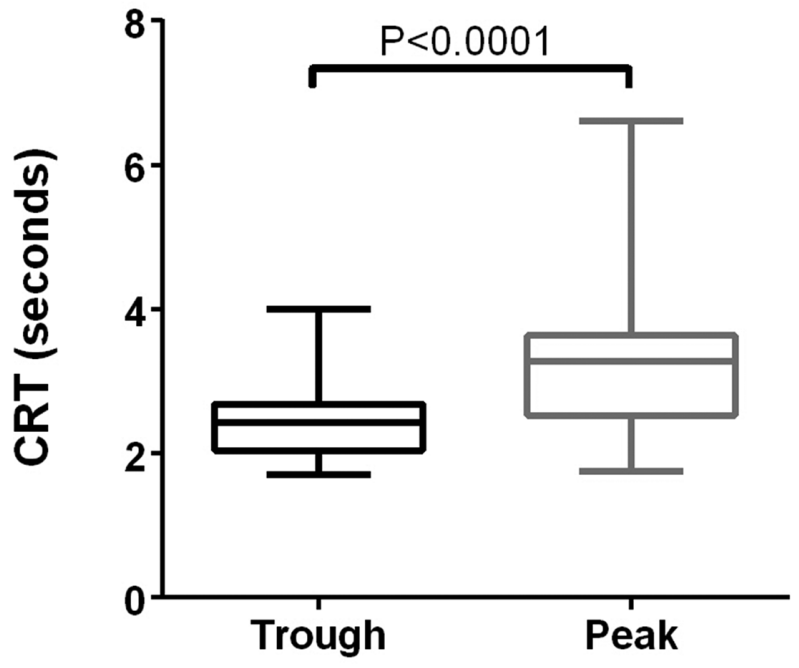

and effectiveness of resuscitation efforts (APLS 2011). CRT is one of the most sensitive predictors of dehydration and bacterial infection in children, and it correlates with markers of end organ perfusion (Tibby 1999, Craig 2010). Automating CRT using photoplethysmography (PPG) with a pressure application system may improve its accuracy and reliability.

Aim Establish if a green light PPG and a pressure application system could detect an increase in CRT after skin cooling.

Methods Healthy children (aged 5-18 years) underwent selective arm cooling as a model of reduced skin blood flow through the microcirculation (Roustit and Cracowski 2013). An automated green light PPG CRT device was attached to their forearm and their arm was then placed into a refrigeration device at $4^{\circ} \mathrm{C}$ for $20 \mathrm{~min}$. Using $7 \mathrm{~min}$ rolling averages, the trough (early phase) and peak (late phase) CRT values were compared. Ethical approval was given.

Results Participants $(n=16)$ demonstrated a significant increase in CRT (as assessed by green PPG) in 15 of the 16 cases (P < 0.001)(see Figure).

Conclusions It is feasible to measure changes in the microcirculation of children using an automated device that mimics CRT testing. Further work in clinical settings is needed to establish if this has clinical utility.

\section{PO-0343 HEMOLYTIC CHARACTERISTICS OF A NEW DIAGONAL PUMP FOR EXTRACORPORAL RESPIRATORY SUPPORT IN NEONATES WITH RESPIRATORY FAILURE}

S Herber-Jonat, K Förster, M Klemme, A Schulze, AW Flemmer. Neonatology Perinatal Centre Großhadern, Dr Von Hauner Childrens'Hospital LMU Munich, Munich, Germany

\subsection{6/archdischild-2014-307384.991}

Background Neonatal extracorporal membrane oxygenation (ECMO) for pulmonary failure is characterised by small cannulas and low flow rates. Novel rotary pumps with diagonal blood flow and a reduced priming volume are now available. Bench studies in different in-vitro models and the short-term use for cardio-pulmonary bypass in paediatric patients are encouraging. However, little data exist on the effect of these devices on hemolysis in the defined group of neonates with respiratory failure.

Design We prospectively studied circuit settings, plasma-free haemoglobin (fHb), lactatdehydrogenase and coagulation activation in neonates receiving veno-arterial ECMO due to respiratory failure with a novel diagonal pump system.

Results Eight newborns (3280 g [2700; 4380, Median; Range]) received veno-arterial ECMO through $8-10 \mathrm{Fr}$ venous and $8 \mathrm{Fr}$ arterial cannulas appropriate for neck vessels. All infants were maintained on a centrifugal pump system for $4.3 \mathrm{~d}(2 ; 7.3)$. Median flow rate and venous cannula pressure were $259 \mathrm{ml} / \mathrm{min}$ $(215 ; 314)$, and $-9 \mathrm{~cm} \mathrm{H} 2 \mathrm{O}(-50 ;-1)$ respectively. The median revolutions per minute of the pump were 4774 (4008; 6801). Mild hemolysis was detected within $24 \mathrm{~h}$ in all patients [median $\mathrm{fHb} 12.1 \mathrm{mg} / \mathrm{dl}(7.0 ; 70.9)]$ with a further increase after $48 \mathrm{hrs}$. Two patients $(25 \%)$ developed disseminated intravascular coagulation and persistent moderate hemolysis.

Conclusion Our in vivo data support previous in vitro studies by demonstrating that the use of a centrifugal pump on small venous and arterial cannula appears to be safe and effective for neonatal ECMO. Yet, ECMO for more than 48 hrs holds the risk for an increased hemolysis and disseminated intravascular coagulation. 